\title{
Changes in Aesthetic Appeal of Tattoos Are Influenced by the Attractiveness of Female Models
}

\author{
Jordan Ball, Robert J. F. Elsner \\ Erskine College, Due West, USA
}

\begin{abstract}
External perceptions of individuals based on the presence of tattoos were assessed. College students completed an online attitudinal assessment in a $4 \times 2$ design. Participants were asked to rate the aesthetics of tattoos and models (11 women and 17 men) from Photoshopped images. Results ( $n=152$ usable of 177 collected) indicate that as whole models and tattoos are not affected in aesthetic appeal, but individually some models and tattoos were affected. Tattoos were rated as better quality art when the model can be seen; thus, contextualization allows for greater appreciation of the tattoo. Results support extant research that indicates women's attractiveness is not affected by tattoos, which also providing a new approach for tattooed individuals.
\end{abstract}

Keywords: tattoos, attraction, aesthetic appeal, body modification

\section{Introduction}

Tattoos are an increasingly popular form of self-expression (Braverman, 2012; Dickson, 2015), and individuals who acquire tattoos do so for a plethora of reasons. Documented reasons include religious expression (Firmin, 2008; Koch \& Roberts, 2012), personal narrative (Wohlrab, Stahl, \& Kappeler, 2007), or even a metaphorical " "patch' to repair holes... and to reconstruct (them)" (Karacaoglan, 2012). Tattoos serve as a method for commemorating a loss, or memorializing a challenging time in one's life that has been overcome.

Contrary to such positive internal motivating factors, tattoos are still socially perceived in a negative light. The general populace views tattoos as a "business faux-pas": Foltz (2014) demonstrated that, when placed in a hypothetical hiring position, even tattooed participants viewed tattoos as a reason to not hire a potential employee. This disconnect in understanding between tattooed individuals stems from the breadth of content and the multitude of reasons for obtaining a tattoo. Williams, Thomas, and Christensen (2014) suggested that this is a high priority issue that social work should tackle head-on since tattoos have the potential to "turn off your clients, colleagues, and administration" (Kirst-Ashman \& Hull, 2015).

However, some research supports the validity of stereotypes against tattoos individuals to a degree. Similar results indicate several key attributes is likely to be found in tattooed individuals: greater alcoholic tendencies (Guéguen, 2012a), earlier onset of sexual activity (Nowosielski, Sipiński, Kuczerawy, Kozłowska-Rup, \& Skrzypulec-Plinta, 2012; Guéguen, 2012b), and increased extraversion and experience-seeking characteristics (Swami, Pietschnig, Bertl, Nader, Stieger, \& Voracek, 2012). Further extant research indicates an increase in deviant behavior among tattooed adolescents (Roberts \& Ryan, 2002), and this

Jordan Ball, B. S., senior research associate, Department of Psychology, Erskine College.

Robert J. F. Elsner, Ph.D., professor, Department of Psychology, Erskine College. 
deviancy continues into college, although there is no link between tattoos and school performance (Horne, 2007; Martin \& Dula, 2010).

Extant research all meets at a vital intersection of attractiveness. While some of the characteristics listed may be deemed as attractive qualities, how are those reconciled with the fact that tattoos are still a faux-pas despite the growing population of tattooed individuals?

Most current research looks only at the potential negative effect that a tattoo has on an individual, or rather, what that tattoo might be able to tell us about that person. The current study was designed conversely, with an intended goal to determine the effect that a tattooed individual has on the perceived quality of that tattoo, therefore: $\mathrm{H}_{1}$ : Attractiveness of female models will alter the aesthetic appeal of a tattoo; and $\mathrm{H}_{2}$ : Attractiveness of female models will be affected by the presence of a tattoo. While hypothesis one is exploratory in nature, hypothesis two is supported by Swami and Furnham (2007), who showed that line drawings of females with tattoos were less physically attractive.

Guéguen (2013) indicated that men rated women as more promiscuous when lower back tattoos were visible; while promiscuity is not necessarily related to attractiveness, it could be a contributing factor. This link also creates a reason for focusing only on women; while some findings discuss promiscuity of males, most focus on the promiscuity of women. Furthermore, most studies focus on participants' views of tattooed women; this experiment will continue in this vein, but will also diverge in how it treats tattooed individuals. Thus far, research on this cohort simply analyzes tattooed individuals as a whole. Can styles of tattoos (e.g., traditional versus new school) combined with the content (tribal versus scripture) really be analyzed as one entity when also taking into account the variability of reasons for obtaining a tattoo? The purpose of this experiment, therefore, was to treat every tattooed individual as just that: an individual.

\section{Method}

\section{Participants}

After receiving Institutional Review Board (IRB-Ethics) approval, a link to the assessment was distributed via social media and campus email. Participants were allowed to complete the assessment at their own leisure. After giving an electronic signature to indicate informed consent, the participant then completed the experiment. Of the 177 initial college student respondents, 152 complete data sets were compiled, identified as Caucasian (80.4\%), African American (3.6\%), Asian (0.7\%), Hispanic (2.0\%), Native American (0.3\%), or other (2.3\%). Of the 152 data sets, only 138 indicated gender: 91 identified as female, and 48 identified as male; $26 \%(n=40)$ of participants indicated that they had at least one tattoo, a sample that is consistent with other findings (Martin, 1997; Roberts \& Ryan, 2002).

\section{Materials}

Attitudinal assessment scale. A series of 50 pictures (33 target/female and 17 distractor/male) were presented with Likert scales. 11 photos of women comprised the target stimuli, and were manipulated using Photoshop to remove a tattoo as well as to zoom in on the tattoo itself. These photos, then, were of three types: each model with a tattoo, each model without a tattoo, and a close up of the tattoo itself (see appendix A for example). Presented in a randomized order, the distractor images were interspersed amongst the target images; the questions for the distractors were the same as for the targets. Men were chosen as distractor images to obfuscate the focus on women, and both tattooed and non-tattooed men were included. 
Closing up pictures of tattoos were rated on the perceived quality on a scale of 1-10, with 1 being "I don't like this tattoo at all" and 10 being "I like this tattoo very much". Perception of quality was determined using personal liking because most participants would not be aware of what factors a professional might use to assess actual quality (e.g., blown out lines, color saturation, line weight). A Likert scale from 1-10 was used to assess the physical attractiveness of models without tattoos, with 1 being "Very unattractive" and 10 being "Very attractive". Photos of models with tattoos were presented with both scales.

\section{Procedure}

Using stock images from a variety of internet sources, the assessment was structured such that each item was answered before moving on to the next item. Each stock image was of a woman with one tattoo that was subsequently removed using Photoshop. The models chosen were Caucasian, and this was done simply because tattoos are most easily seen on paler skin. From each image, two additional photos were created: the model without the tattoo, and a close up of the tattoo. Questions were paired with each picture as described above in the materials section. After completing the assessment, participants were asked demographic questions that included additional questions related to tattoos. Participants were asked if anyone in their immediate family had a tattoo. Participants were also asked if they themselves had a tattoo, and if so, how many (see Appendix A for examples of photos).

\section{Results}

All results were compiled and analyzed using SPSS (v. 23, IBM Corp, Armonk, NY, USA). From a broad perspective, a repeated measure ANOVA indicated that presence of a tattoo did not change the overall attractiveness of models. Despite this, a major thrust of this experiment was to determine if individual differences arose, so further tests were run on each individual model. Paired samples $t$-tests were run on each of the 11 models to differentiate between their attractiveness with a tattoo and without a tattoo. Not all of these were significant, but the $t$-tests indicated that models $1(t(140)=-2.323, p<0.022)$ and $2(t(143)=-2.292, p<$ 0.023 ) both decreased in attraction with the presence of a tattoo. While some models increased in attraction, most decreased, but these changes were not significant. Paired samples $t$-tests for aesthetic appeal of tattoos yielded both positive and negative fluctuation. For example, one tattoo increased in aesthetic appeal when the model could be seen, $t(144)=-7.308, p<0.0001$, but the aesthetic appeal of another decreased, $t(151)=3.561$, $p<0.0001$. There was a difference in rating of attraction between gender for both of these models, $F(1,136)=$ $14.824, p<0.0001$ for the former and $F(1,136)=4.065, p<0.046$ for the latter.

An "a postieri" repeated measures analysis conducted on tattooed participants and their perception of attraction of models with and without tattoos showed that there was a significant difference between tattooed individuals with different numbers of tattoos (those that had 1, 2, 3, and 4 tattoos), $F(3,36)=3.217, p<0.034$. A Least Significant Difference (LSD) post hoc test indicated none of these groups significantly differed from one another; however, the participants with 3 and 4 tattoos approached significance $(p<0.074)$.

Secondary analysis showed that there was a positive correlation between having family members who have a tattoo and whether or not the participant also had a tattoo, $r=0.324, n=137, p<0.001$.

\section{Discussion}

This study refines earlier findings that indicate physical attractiveness decreases when a tattoo is present (Swami \& Furnham, 2007; Resenhoeft, 2008). While it is true that many models decreased in physical 
attractiveness, this decrease was not significant; only two models significantly decreased in attractiveness, which supports treating tattooed individuals on an individual basis. These results align more with those found by Seiter and Hatch (2005), who determined that a model with a tattoo was not significantly impacted in terms of physical attractiveness.

Tattoo location is an integral factor in making judgments on other individuals as indicated by studies concerning facial tattoos (Funk \& Todorov, 2013; Swami, Stieger, Pietschnig, Voracek, Furnham, \& Tovee, 2012); location was not directly assessed here, but some inferences towards placement can still be made. For the few models that did experience a slight increase in attractiveness with a tattoo, these models had their tattoo placed in provocative areas. From an evolutionary perspective, these tattoos serve as another tactic used by women to draw attention to particular assets of the body (Greer \& Buss, 1994). This idea should be elaborated on in future studies.

Further evidence that the tattooed population should be treated on an individual basis arises when questions of content are considered. In this study, two models had the same content that was similar in overall quality. While both models experienced a slight decrease in attractiveness, the aesthetic appeal of one tattoo increased while the other decreased. The tattoo that increased in aesthetic appeal was on an attractive model, while the tattoo that decreased in appeal was on an unattractive model. A favorable outlook of a tattoo may lead to a favorable outlook of that person, causing preferential treatment of the person if the tattoo is pleasing.

All but one tattoo increased in terms of aesthetic appeal when the model could be seen, this tattoo was on an unattractive model, and an argument could be made that the unattractive model negatively influenced the aesthetic appeal of the tattoo, while attractive models increased the appeal of the respective tattoo.

Past research shows that having a tattoo alters your perception of other tattooed individuals (Koch, Roberts, Armstrong, \& Owen, 2010), and these results concur. However, while not statistically significant, post hoc tests during this experiment honed this idea, providing evidence that the only distinction between tattooed individuals arises from those participants who have three and four tattoos. Should this experiment have more tattooed participants, perhaps the results would have yielded significance. Past research has been conducted on the personality differences in individuals with different numbers of tattoos (Owen, Armstrong, Koch, \& Roberts, 2013; Koch, Roberts, Armstrong, \& Owen, 2015); this experiment dismisses those differences in favor of a behavioral approach that shows that, despite the personality differences, the tattooed individuals are more alike than previously assumed. For tattooed individuals, correlations did show that family members have an influence on obtaining a tattoo, which increased slightly since the correlations established recently by Roberts, Koch, Armstrong, and Owen (2006).

Due to the focus of treating tattooed individuals on an individual basis, the stimuli were chosen from a naturalistic perspective rather than attempting to standardize exposure stimuli using line drawings that have little relation to the real three-dimensional world in which we live; because of this, extraneous factors that were not controlled for, are still shown to have impact on rating of attractiveness, include: clothing appearance (Guéguen, 2011), cosmetics (Jacob, Guéguen, Boulbry, \& Ardicioni, 2009), hair color (Guéguen \& Lamy, 2009), posture, lighting, and amount of skin shown by each model. These variables could be contributing factors that explain the differences in physical attractiveness of models. Furthermore, an argument could be made that, in light of these aforementioned factors, the perceptual impact of a tattoo is negligible in comparison.

Further research on this topic should seek to extrapolate results to male models and models of different ethnicities, as well as control for the limitations listed above. More emphasis should also be put on determining 
the extent to which tattooed individuals are affected by the size, content, and location of the tattoo. Also, models in this study only had one tattoo. Studies such as those listed previously have indicated that there is a difference in tattooed individuals for those that have more than four tattoos as compared to those that have less than four tattoos, so perhaps there is a perceptual difference of these individuals as well. Considerations should also be given to color versus black and white tattoos, which no study as of yet has sought to address.

\section{References}

Braverman, S. (2012). One in five U.S. adults now has a tattoo. Harris Polls. Retrieved from http://www. harrisinteractive.com/vault/Harris\%20Poll\%2022\%20 Tattoos?2.23.12.pdf

Dickson, L. N. (2015). To ink or not to ink: The meaning of tattoos among college students. College Student Journal, 49, 106-120.

Firmin, M. T. (2008). Christian student perceptions of body tattoos: A qualitative analysis. Journal of Psychology \& Christianity, 27, 195-204.

Foltz, K. A. (2014). The millennia's perception of tattoos: Self-expression of business faux pas? College Student Journal, 48, 589-602.

Funk, F., \& Todorov, A. (2013). Criminal stereotypes in the courtroom: Facial tattoos affect guilt and punishment differently. Psychology, Public Policy, and Law, 19, 466-478.

Greer, A. E., \& Buss, D. M. (1994). Tactics for promoting sexual encounters. Journal of Sex Research, 31, 185-201.

Guéguen, N. (2011). The effect of women's suggestive clothing on men's behavior and judgment: A field study. Psychological Reports, 109, 635-638.

Guéguen, N. (2012a). Tattoos, piercings, and alcohol consumption. Alcoholism: Clinical and Experimental Research, 36, $1253-1256$.

Guéguen, N. (2012b). Tattoos, piercings, and sexual activity. Social Behavior \& Personality: An International Journal, 40, 1543-1547.

Guéguen, N. (2013). Effects of a tattoo on men's behavior and attitudes towards women: An experimental field study. Archives of Sexual Behavior, 42, 1517-1524.

Guéguen, N., \& Lamy, L. (2009). Hitchhiking women's hair color. Perceptual and Motor Skills, 109, 941-948.

Horne, J. E. (2007). Tattoos and piercings: Attitudes, behaviors, and interpretations of college students. College Student Journal, 41, 1011-1020.

IBM Corp. (2014). IBM SPSS statistics for windows, version 23.0. Armonk, NY: IBM Corp.

Jacob, C., Guéguen, N., Boulbry, G., \& Ardicioni, R. (2009). Waitresses' facial cosmetics and tipping: A field experiment. International Journal of Hospitality Management, 29, 188-190.

Karacaoglan, U. (2012). Tattoo and taboo: On the meaning of tattoos in the analytic process. The International Journal of Psychoanalysis, 93, 5-28.

Kirst-Ashman, K. K., \& Hull, G. H. Jr. (2015). Generalist practice with organizations and communities (6th ed.). Stamford, CT: Cengage Learning.

Koch, J. R., \& Roberts, A. E. (2012). The protestant ethic and the religious tattoo. The Social Science Journal, 49, $210-213$.

Koch, J. R., Roberts, A. E., Armstrong, M. L., \& Owen, D. C. (2010). Body art, deviance, and American college students. Social Science Journal, 47, 151-161.

Koch, J. R., Roberts, A. E., Armstrong M. L., \& Owen, D. C. (2015). Tattoos, gender, and well-being among American college students. The Social Science Journal, 52, 536-541.

Martin, A. (1997). On teenagers and tattoos. Journal of the American Academy of Child and Adolescent Psychiatry, 36, 860-861.

Martin, B. A., \& Dula, C. S. (2010). More than skin deep: Perceptions of, and stigma against, tattoos. College Student Journal, 44, 200-206.

Nowosielski, K., Sipiński, A., Kuczerawy, I., Kozłowska-Rup, D., \& Skrzypulec-Plinta, V. (2012). Tattoos, piercing, and sexual behaviors in young adults. Journal of Sexual Medicine, 9, 2307-2314.

Owen, D. C., Armstrong, M. L., Koch, J. R., \& Roberts, A. E. (2013). College students with body art: Well being or higher risk behavior? Journal of Psychosocial Nursing, 51, 20-28.

Resenhoeft, A. D. (2008). Tattoos can harm perceptions: A study and suggestions. Journal of American College Health, 56, 593-596. 
Roberts, T. A., \& Ryan, S. A. (2002). Tattooing and high-risk behavior in adolescents. Pediatrics, 110, 1058-1063.

Roberts, A. E., Koch, J. R., Armstrong, M. L, \& Owen, D. C. (2006). Correlates of tattoos and reference groups. Psychological Reports, 99, 933-934.

Seiter, J. S., \& Hatch, S. (2005). Effect of tattoos on perceptions of credibility and attractiveness. Psychological Reports, 96, 1113-1120.

Swami, V., \& Furnham, A. (2007). Unattractive, promiscuous, and heavy drinkers: Perceptions of women with tattoos. Body Image, 4, 343-352.

Swami, V., Pietschnig, J., Bertl, B., Nader, I. W., Stieger, S., \& Voracek, M. (2012). Personality differences between tattooed and non-tattooed individuals. Psychological Reports, 111, 97-106.

Swami, V., Stieger, S., Pietschnig, J., Voracek, M., Furnham, A., \& Tovee, M. J. (2012). The influence of facial piercings and observer personality on perceptions of physical attractiveness and intelligence. European Psychologist, 17, 213-221.

Williams, D. J., Thomas, J., \& Christensen, C. (2014). "You need to cover your tattoos!”: Reconsidering standards of professional appearance in social work. Social Work, 59, 373-375.

Wohlrab, S., Stahl, J., \& Kappeler, P. M. (2007). Modifying the body: Motivations for getting tattooed and pierced. Body Image, 4 , $87-95$.

\section{Appendix A: Example of the Stimuli Used.}
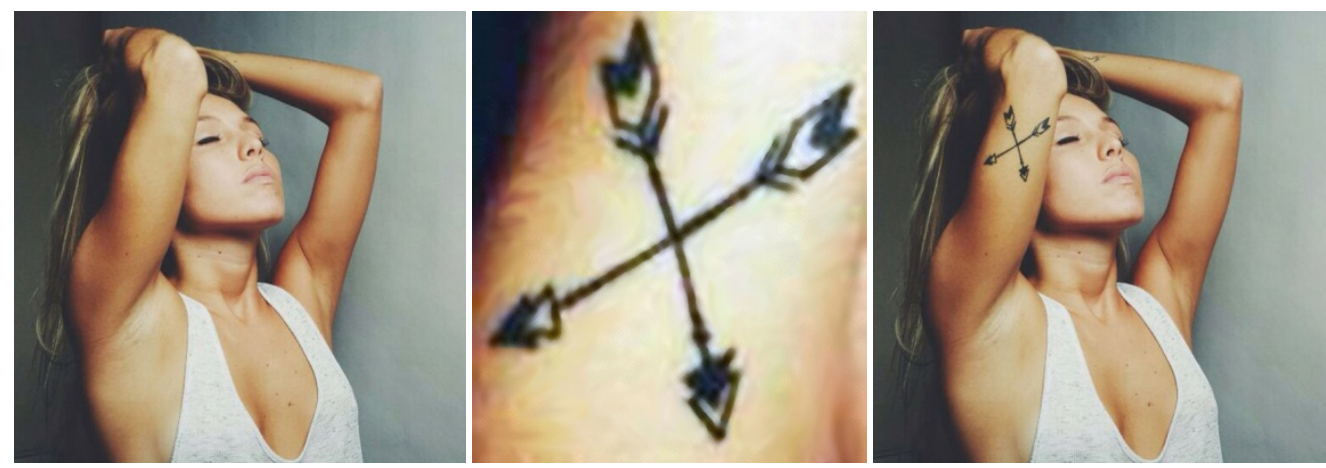

Figure 1. Model 9 and Tattoo 7. The model with the tattoo was the original picture, and the two preceding photos were created using Photoshop. 